implicit hazards associated with the boat maneuvering and exposure to the elements. Nonetheless, there have been significant tragedies. Recent improvements in safety gear and advances in technology have allowed for more acute medical management of sailors.

Objective.-This study investigates the relative frequency of injuries and illnesses sustained during offshore sailboat racing in the Great Lakes, which has never been reported. These results will be compared with those published in a study that investigated the same occurrences during the Newport Bermuda ocean race to determine whether there is a difference in frequency or type found on the open ocean versus the Great Lakes.

Methods.-The study design was prospective, descriptive, and observational. Surveys were self-reported and collected after the race from the boat representative. De-identified data were entered into a Microsoft Excel database and analyzed by a professional statistician.

Results.-In 2013, representatives of 164 boats responded to the survey in one offshore race, which represented 1260 sailors; 146 illnesses were reported, and 9 boats reported at least 1 injury. In 2014, representatives of 435 boats responded to the survey in two offshore races, which represented 3525 sailors. The 2014 data are still being analyzed.

Conclusions.-The 2013 rate of injury and illnesses in the Great Lakes was significant at 125 per 1000 races per sailor when compared with the Newport Bermuda race at only 12 . Injuries in the form of contusions were most common in the Great Lakes races as opposed to lacerations in the ocean races, and seasickness was the most common illness in both environments.

Thomas Kopp, MD

David Ledrick, MD

Julie Stausmire, MSN

Kari Stausmire, BA

Toledo, OH, USA

\section{Paper Tape Prevents Foot Blisters: Randomized Prevention Trial Assessing Paper Tape in Endurance Distances II (Pre-TAPED II)}

Background.-Friction foot blisters, a common and potentially disabling injury, have been found to be the most likely factor adversely affecting ultramarathon performance. An inexpensive and effective prevention technique is needed for this ubiquitous outdoor occurrence.

Objective.-To determine whether paper tape could prevent friction foot blisters on blister prone areas of multistage ultramarathoners.

Methods.-This multisite prospective randomized trial was undertaken during the 2014 RacingThePlanet, a 155-mile, 6-stage ultramarathon in the Jordan, Gobi, Madagascar, and Atacama deserts. Paper tape was applied to a randomly selected foot before the race, either to participants' blister prone areas or to a randomly selected spot, with untaped areas of the same foot as control. Study outcome was a blister anywhere on the study foot.

Results.-In all, 128 participants were enrolled, and 109 (85.2\%) were compliant. There were 31 women $(22.5 \%)$, with a mean age of 39.3 years (range 22 to 63 years) and body mass index (BMI) of $24.2 \mathrm{~kg} / \mathrm{m}^{2}$ (range 17.4 to $35.1 \mathrm{~kg} / \mathrm{m}^{2}$ ). Intentto-treat analysis revealed 106 participants (83\%) had 117 blisters, with treatment success in 98 runners (77\%). Paper tape reduced blisters by $40 \%$ ( $P<.01,95 \%$ CI: 28 to 52 ), and number needed to treat, 1.31. Results were similar in the compliant group $(\mathrm{NNT}=1.35)$. The majority of participants (78\%) had 1 blister, most commonly on the toes $(\mathrm{n}=58,50 \%)$ and heel $(n=27,23 \%)$, and 94 blisters $(80 \%)$ occurred by the end of stage 2 of the race. Treatment success and number of blisters were significantly associated with hours of exposure $(P=.01)$ and stage of race $(P=.02)$; BMI correlated with number of blisters ( $r=0.23, P<.01,95 \% \mathrm{CI}$ : 0.06 to 0.39 ).

Conclusions.-Paper tape was found to have robust protective effect against foot blisters for multistage ultramarathon runners.

Grant S. Lipman, MD

Mark Christensen, DO

Alexandra DiTullio, MD

Katherine Shea, MD

Palo Alto, CA, USA

Louis J. Sharp, MD

Andrew Dalton, DO

Chicago, IL, USA

Caleb Phillips, $\mathrm{PhD}$

Boulder, CO, USA

Pearlly Ng, MD

Salt Lake City, UT, USA

Jennifer Shangkuan, MD

New York, NY, USA

Brian J. Krabak, MD

Seattle, WA, USA

\section{"Out of the Box" Disaster Training: Low-Cost Training Tools Improve Medical Team Disaster Performance}

Background.- The provision of life-saving medical care in austere environments requires rescue team training in a simulated setting. Not every rescue organization has the capability to train in the field owing to weather, limited budgets, or schedule constraints.

Objective.-We developed a competency-based curriculum for patient care providers using an indoor, easily reproducible, 